Bull. Korean Math. Soc. 45 (2008), No. 4, pp. 763-780

\title{
OSTROWSKI TYPE INEQUALITY FOR ABSOLUTELY CONTINUOUS FUNCTIONS ON SEGMENTS IN LINEAR SPACES
}

\author{
Eder Kikianty, Sever S. Dragomir, and Pietro Cerone
}

\begin{abstract}
An Ostrowski type inequality is developed for estimating the deviation of the integral mean of an absolutely continuous function, and the linear combination of its values at $k+1$ partition points, on a segment of (real) linear spaces. Several particular cases are provided which recapture some earlier results, along with the results for trapezoidal type inequalities and the classical Ostrowski inequality. Some inequalities are obtained by applying these results for semi-inner products; and some of these inequalities are proven to be sharp.
\end{abstract}

\section{Introduction}

In 1938, A. Ostrowski (see [25, p. 226]) considered the problem of estimating the deviation of a function from its integral mean. For any continuous function $f$ on $[a, b] \subset \mathbb{R}$ which is differentiable on $(a, b)$ and $\left|f^{\prime}(x)\right| \leq M$ for all $x \in(a, b)$, the inequality

$$
\left|f(x)-\frac{1}{b-a} \int_{a}^{b} f(y) d y\right| \leq\left[\frac{1}{4}+\frac{\left(x-\frac{a+b}{2}\right)^{2}}{(b-a)^{2}}\right](b-a) M,
$$

holds for every $x \in[a, b]$ (see [25, pp. 226-227] for the complete proof). This is then known as the Ostrowski inequality (see [24, p. 468]). The first factor on the right hand side of (1.1) reaches the value of $\frac{1}{4}$ at the midpoint and monotonically increases to $\frac{1}{2}$ which is attained at both endpoints [25, p. 226]. It implies that the constant $\frac{1}{4}$ is best possible, that is, it cannot be replaced by a smaller quantity (see also [2, pp. 3775-3776], for an alternative proof).

The Ostrowski inequality has been generalised for functions of bounded variations (see [15, p. 374] and [19, pp. 3-4]). For this class of functions, the results have been developed to estimate the absolute difference between the linear combination of values of a function at $k+1$ partition points (of a closed interval) from its integral mean (see [10]). A similar result has been obtained for the

Received March 21, 2008.

2000 Mathematics Subject Classification. 26D15, 46C50.

Key words and phrases. Ostrowski type inequality, absolutely continuous function, semiinner product. 
class of absolutely continuous functions (see $[11,12,15,17,19]$ ). The classical Ostrowski inequality and the trapezoidal type inequality are obtained by considering some particular cases of the generalised Ostrowski type inequality (see [15, pp. 378-381]).

Another possibility of generalising the Ostrowski inequality is to consider the case of convex functions. Since any convex function is locally Lipschitzian (hence, it is locally absolutely continuous), thus it can be connected to the previous mentioned cases (see [15, 18]). For other possible directions, we refer to the results in $[4,5,6,7,8,9]$.

An extension of the Ostrowski inequality to functions with values in Banach spaces has been given in [3]. A similar result has been established for functions defined on segments in linear space (see [17]). An application for semi-inner products in any normed linear spaces was also provided in [17, pp. 95-99]. However, the sharpness for the constants of these inequalities has not been considered.

In this paper, we develop an Ostrowski type inequality for estimating deviation of the integral mean of an absolutely continuous function and the linear combination of its values at $k+1$ partition points on a segment in (real) linear spaces. We also provide some particular cases which recapture the results in [17] along with the results for trapezoidal type inequalities and the classical Ostrowski inequality. In a normed linear spaces, we obtain inequalities for semi-inner products by applying the obtained results and these inequalities are more general than those in [17]. Some of these inequalities are proven to be sharp and the proof also covers the sharpness of those in [17].

\section{Definitions}

All definitions and notation which will be used in the paper, are described in this section for references. Throughout this paper, we assume that all linear spaces are over the field of real numbers.

Let $X$ be a linear space and $x, y \in X$. We consider the Gâteaux lateral derivatives of a function $f$ at $x \in X$, as

$$
\left(\nabla_{ \pm} f(x)\right)(y):=\lim _{t \rightarrow 0^{ \pm}} \frac{f(x+t y)-f(x)}{t},
$$

if the above limits exist for any $y \in X$.

Let $x, y \in X, x \neq y$ and define the segment $[x, y]:=\{(1-t) x+t y, t \in[0,1]\}$. Let $f:[x, y] \rightarrow \mathbb{R}$ and the associated function $h=g(x, y):[0,1] \rightarrow \mathbb{R}, h(t)=$ $g(x, y)(t):=f[(1-t) x+t y], t \in[0,1]$. It is well known that the function $h$ is absolutely continuous on $[0,1]$ if and only if $h$ is differentiable almost everywhere, the derivative $h^{\prime}$ is Lebesgue integrable, and $h(t)=\int_{0}^{t} h^{\prime}(s) d s+$ $h(0)$ (see [1, p. 263] and [28, pp. 106-107]).

Lemma 1. With the above notation, $h$ is absolutely continuous if and only if $f$ satisfies the following properties 
(1) $\nabla f[(1-\cdot) x+\cdot y](y-x)$ exists almost everywhere on $[0,1]$;

(2) $\nabla f[(1-\cdot) x+\cdot y](y-x)$ is Lebesgue integrable on $[0,1]$;

(3) $f[(1-t) x+t y]=\int_{0}^{t} \nabla f[(1-s) x+s y](y-x) d s+f(x)$.

Definition 1. Let $f$ be a real-valued function defined on a segment $[x, y]$ of a linear space $X$. We say that $f$ is absolutely continuous on segment $[x, y]$ if $f$ satisfies the conditions (1)-(3) of Lemma 1.

By Definition 1 and Lemma 1 , we conclude that $f$ is absolutely continuous on segment $[x, y]$ if and only if $h$ is absolutely continuous on $[0,1]$.

Assume that $(X,\|\cdot\|)$ is a normed linear space. The function $f_{0}(x)=\frac{1}{2}\|x\|^{2}$ $(x \in X)$ is convex and the following limits

$$
\langle x, y\rangle_{s(i)}:=\left(\nabla+(-) f_{0}(y)\right)(x)=\lim _{t \rightarrow 0^{+(-)}} \frac{\|y+t x\|^{2}-\|y\|^{2}}{2 t},
$$

exist for any $x, y \in X$. They are called the superior (inferior) semi-inner products associated to the norm $\|\cdot\|$ (see [16, pp. 27-39] for further properties).

Throughout this paper, we are also interested in the function: $f_{r}(x)=\|x\|^{r}$ $(x \in X$ and $1 \leq r<\infty)$, which is also a convex function. Therefore, the following limits, which are related to superior (inferior) semi-inner products,

$$
\left(\nabla+(-) f_{r}(y)\right)(x)=\lim _{t \rightarrow 0^{+(-)}} \frac{\|y+t x\|^{r}-\|y\|^{r}}{t}=r\|y\|^{r-2}\langle x, y\rangle_{s(i)},
$$

exist for all $x, y \in X$ whenever $r \geq 2$; otherwise, they exist for any $x \in X$ and nonzero $y \in X$.

\section{The results}

Our main result (Theorem 1) is an Ostrowski type inequality for estimating deviation of the integral mean of an absolutely continuous function and the linear combination of its values at $k+1$ partition points on a segment of a linear space. This result is basically a follow-up for the previous results:

(1) Ostrowski type inequality for estimating the absolute difference between the linear combination of values of a function at $k+1$ partition points from its integral mean (see [10]) ;

(2) Ostrowski type inequality for functions defined on segments of a linear space (see [17]);

(3) Ostrowski inequality for absolutely continuous function (see [11, 12, 15, 17, 19]).

Following Theorem 1, we have two corollaries, in which we consider its particular cases. Corollary 1 estimates the trapezoid type functionals, while Corollary 2 estimates the Ostrowski type functionals. Proofs for these results are provided in Section 4. The results are as follows: 
Theorem 1. Let $X$ be a linear space, $I_{k}: 0=s_{0}<s_{1}<\cdots<s_{k-1}<s_{k}=1$ be a division of the interval $[0,1]$ and $\alpha_{i}(i=0, \ldots, k+1)$ be $k+2$ points such that $\alpha_{0}=0, \alpha_{i} \in\left[s_{i-1}, s_{i}\right](i=1, \ldots, k)$ and $\alpha_{k+1}=1$. If $f:[x, y] \subset X \rightarrow \mathbb{R}$ is absolutely continuous on segment $[x, y]$, then we have

$$
\begin{aligned}
& \left|\int_{0}^{1} f[(1-t) x+t y] d t-\sum_{i=0}^{k}\left(\alpha_{i+1}-\alpha_{i}\right) f\left[\left(1-s_{i}\right) x+s_{i} y\right]\right| \\
& \leq\left\{\begin{array}{l}
{\left[\frac{1}{4} \sum_{i=0}^{k-1} h_{i}^{2}+\sum_{i=0}^{k-1}\left(\alpha_{i+1}-\frac{s_{i}+s_{i+1}}{2}\right)^{2}\right]\|\nabla f[(1-\cdot) x+\cdot y](y-x)\|_{\infty},} \\
\text { if } \nabla f[(1-\cdot) x+\cdot y](y-x) \in L_{\infty}[0,1] ; \\
\frac{1}{(q+1)^{\frac{1}{q}}}\left[\sum_{i=0}^{k-1}\left[\left(\alpha_{i+1}-s_{i}\right)^{q+1}+\left(s_{i+1}-\alpha_{i+1}\right)^{q+1}\right]\right]^{\frac{1}{q}}\|\nabla f[(1-\cdot) x+\cdot y](y-x)\|_{p}, \\
\text { if } \nabla f[(1-\cdot) x+\cdot y](y-x) \in L_{p}[0,1], p>1, \frac{1}{p}+\frac{1}{q}=1 ; \\
{\left[\frac{1}{2} \nu(h)+\max _{i \in\{0, \ldots, k-1\}}\left|\alpha_{i+1}-\frac{s_{i}+s_{i+1}}{2}\right|\right]\|\nabla f[(1-\cdot) x+\cdot y](y-x)\|_{1},}
\end{array}\right.
\end{aligned}
$$

where $\nu(h):=\max \left\{h_{i} \mid i=0, \ldots, k-1\right\}, h_{i}:=s_{i+1}-s_{i}(i=0, \ldots, k-1)$ and $\|\cdot\|_{p}(p \in[1, \infty])$ are the Lebesgue norms.

The constants $\frac{1}{4}, \frac{1}{(q+1)^{\frac{1}{q}}}$ and $\frac{1}{2}$ are sharp.

Corollary 1. Let $X$ be a linear space, $x, y \in X, x \neq y$ and $f:[x, y] \subset X \rightarrow \mathbb{R}$ be an absolutely continuous function on segment $[x, y]$. Then for any $s \in[0,1]$ we have the inequalities

$$
\begin{aligned}
& \left|\int_{0}^{1} f[(1-t) x+t y] d t-s f(x)-(1-s) f(y)\right| \\
& \leq\left\{\begin{array}{c}
{\left[\frac{1}{4}+\left(s-\frac{1}{2}\right)^{2}\right]\|\nabla f[(1-\cdot) x+\cdot y](y-x)\|_{\infty},} \\
\text { if } \nabla f[(1-\cdot) x+\cdot y](y-x) \in L_{\infty}[0,1] \\
\frac{1}{(q+1)^{\frac{1}{q}}}\left[s^{q+1}+(1-s)^{q+1}\right]^{\frac{1}{q}}\|\nabla f[(1-\cdot) x+\cdot y](y-x)\|_{p}, \\
\text { if } \nabla f[(1-\cdot) x+\cdot y](y-x) \in L_{p}[0,1], p>1, \frac{1}{p}+\frac{1}{q}=1 \\
{\left[\frac{1}{2}+\left|s-\frac{1}{2}\right|\right]\|\nabla f[(1-\cdot) x+\cdot y](y-x)\|_{1},}
\end{array}\right.
\end{aligned}
$$

where $\|\cdot\|_{p}(p \in[1, \infty])$ are the usual Lebesgue norms on $L_{p}[0,1]$. Particularly, we have

$$
\begin{aligned}
& \left|\int_{0}^{1} f[(1-t) x+t y] d t-\frac{f(x)+f(y)}{2}\right| \\
& \leq\left\{\begin{array}{l}
\frac{1}{4}\|\nabla f[(1-\cdot) x+\cdot y](y-x)\|_{\infty}, \\
\frac{1}{2(q+1)^{\frac{1}{q}}}\|\nabla f[(1-\cdot) x+\cdot y](y-x)\|_{p}, \quad p>1, \frac{1}{p}+\frac{1}{q}=1 ; \\
\frac{1}{2}\|\nabla f[(1-\cdot) x+\cdot y](y-x)\|_{1} .
\end{array}\right.
\end{aligned}
$$


The constants in (3.2) and (3.3) are sharp.

Remark 1. If $f$ is convex in (3.3), then $\left(\frac{f(x)+f(y)}{2}-\int_{0}^{1} f[(1-t) x+t y] d t\right)$ is non-negative by Hermite-Hadamard integral inequality (see [14, p. 2]).

Corollary 2. Under the assumptions of Corollary 1, we have the following inequalities for any $s \in[0,1]$

$$
\begin{aligned}
& \left|\int_{0}^{1} f[(1-t) x+t y] d t-f[(1-s) x+s y]\right| \\
& \leq\left\{\begin{array}{c}
{\left[\frac{1}{4}+\left(s-\frac{1}{2}\right)^{2}\right]\|\nabla f[(1-\cdot) x+\cdot y](y-x)\|_{\infty},} \\
\text { if } \nabla f[(1-\cdot) x+\cdot y](y-x) \in L_{\infty}[0,1] ; \\
\frac{1}{(q+1)^{\frac{1}{q}}\left[s^{q+1}+(1-s)^{q+1}\right]^{\frac{1}{q}}\|\nabla f[(1-\cdot) x+\cdot y](y-x)\|_{p},} \\
\text { if } \nabla f[(1-\cdot) x+\cdot y](y-x) \in L_{p}[0,1], p>1, \frac{1}{p}+\frac{1}{q}=1 ; \\
{\left[\frac{1}{2}+\left|s-\frac{1}{2}\right|\right]\|\nabla f[(1-\cdot) x+\cdot y](y-x)\|_{1},}
\end{array}\right.
\end{aligned}
$$

where $\|\cdot\|_{p}(p \in[1, \infty])$ are the usual Lebesgue norms on $L_{p}[0,1]$. Particularly, we have

$$
\begin{aligned}
& \left|\int_{0}^{1} f[(1-t) x+t y] d t-f\left(\frac{x+y}{2}\right)\right| \\
\leq & \left\{\begin{array}{l}
\frac{1}{4}\|\nabla f[(1-\cdot) x+\cdot y](y-x)\|_{\infty}, \\
\frac{1}{2(q+1)^{\frac{1}{q}}\|\nabla f[(1-\cdot) x+\cdot y](y-x)\|_{p}, \quad p>1, \frac{1}{p}+\frac{1}{q}=1 ;} \\
\frac{1}{2}\|\nabla f[(1-\cdot) x+\cdot y](y-x)\|_{1} .
\end{array}\right.
\end{aligned}
$$

The constants in (3.4) and (3.5) are sharp.

Remark 2. If $f$ is convex in (3.5), then $\left(\int_{0}^{1} f[(1-t) x+t y] d t-f\left(\frac{x+y}{2}\right)\right)$ is non-negative by Hermite-Hadamard integral inequality (see [13, p. 2]).

Remark 3. The inequality (3.5) has been obtained in [17, Corollary 1]. We also note that the bounds in (3.4) and (3.5) are the same as the ones in (3.2) and (3.3), respectively. Cerone in [6, Remark 1] stated that there is a strong relationship between the Ostrowski $((3.4)$ and (3.5)) and trapezoidal functionals $((3.2)$ and (3.3)) which is highlighted by the symmetric transformations amongst their kernels. Particularly, the bounds in the Ostrowski and trapezoidal type inequalities are the same [6, p. 317]. Therefore, from now on, we will only present the results for the Ostrowski functionals. As for the proof of the best constants, the same choices of vectors and linear spaces would also apply for the trapezoidal functionals, unless told otherwise.

Example 1 (Example of a non convex function). Let $(X,\|\cdot\|)$ be a normed linear space and consider the absolutely continuous function $f(x)=\ln (\|x\|)$, 
$x \in X \backslash\{0\}$. Applying this to (3.5) we obtain the following for any linearly independent $x, y \in X$ :

$$
\begin{aligned}
\leq & \left.\left|\int_{0}^{1} \ln (\|(1-t) x+t y\|) d t-\ln \left(\left\|\frac{x+y}{2}\right\|\right)\right|\right) \\
\leq & \left\{\begin{array}{l}
\frac{1}{4} \sup _{u \in[0,1]}\left|\frac{\langle y-x,(1-u) x+u y\rangle_{s(i)}}{\|(1-u) x+u y\|^{2}}\right|, \\
\frac{1}{2(q+1)^{\frac{1}{q}}}\left(\int_{0}^{1}\left|\frac{\langle y-x,(1-u) x+u y\rangle_{s(i)}}{\|(1-u) x+u y\|^{2}}\right|^{p} d u\right)^{\frac{1}{p}}, p>1, \frac{1}{p}+\frac{1}{q}=1 ; \\
\frac{1}{2} \int_{0}^{1}\left|\frac{\langle y-x,(1-u) x+u y\rangle_{s(i)}}{\|(1-u) x+u y\|^{2}}\right| d u,
\end{array}\right.
\end{aligned}
$$

by (2.1) and using the chain rule. Using the Cauchy-Schwarz inequality for superior (inferior) semi-inner products (see [16, p. 29]), we obtain

$$
\begin{aligned}
& \left|\int_{0}^{1} \ln (\|(1-t) x+t y\|) d t-\ln \left(\left\|\frac{x+y}{2}\right\|\right)\right| \\
\leq & \|y-x\|\left\{\begin{array}{l}
\frac{1}{4} \sup _{u \in[0,1]}\|(1-u) x+u y\|^{-1}, \\
\frac{1}{2(q+1)^{\frac{1}{q}}}\left(\int_{0}^{1}\|(1-u) x+u y\|^{-p} d u\right)^{\frac{1}{p}}, p>1, \frac{1}{p}+\frac{1}{q}=1 ; \\
\frac{1}{2} \int_{0}^{1}\|(1-u) x+u y\|^{-1} d u .
\end{array}\right.
\end{aligned}
$$

\section{Proof of main results}

Proof of Theorem 1. Under the assumptions, we have the Ostrowski type inequality for absolutely continuous function $h(\cdot)$ that has been established in $[10,11,12,15]$

$$
\begin{aligned}
& \left|\int_{a}^{b} h(t) d t-\sum_{i=0}^{k}\left(\alpha_{i+1}-\alpha_{i}\right) h\left(s_{i}\right)\right| \\
& \leq\left\{\begin{array}{l}
{\left[\frac{1}{4} \sum_{i=0}^{k-1} h_{i}^{2}+\sum_{i=0}^{k-1}\left(\alpha_{i+1}-\frac{s_{i}+s_{i+1}}{2}\right)^{2}\right]\left\|h^{\prime}\right\|_{\infty}, \quad \text { if } h^{\prime} \in L_{\infty}[a, b] ;} \\
\frac{1}{(q+1)^{\frac{1}{q}}}\left[\sum_{i=0}^{k-1}\left[\left(\alpha_{i+1}-s_{i}\right)^{q+1}+\left(s_{i+1}-\alpha_{i+1}\right)^{q+1}\right]\right]^{\frac{1}{q}}\left\|h^{\prime}\right\|_{p}, \\
\text { if } h^{\prime} \in L_{p}[a, b], p>1, \frac{1}{p}+\frac{1}{q}=1 ; \\
{\left[\frac{1}{2} \nu(h)+\max _{i \in\{0, \ldots, k-1\}}\left|\alpha_{i+1}-\frac{s_{i}+s_{i+1}}{2}\right|\right]\left\|h^{\prime}\right\|_{1} .}
\end{array}\right.
\end{aligned}
$$

Consider the auxiliary function $h(t)=g(x, y)(t)=f[(1-t) x+t y]$ defined on $[0,1]$. Since $f$ is absolutely continuous on the segment $[x, y]$, it follows that $h=g(x, y)$ is an absolutely continuous function and we may apply the above inequality. We obtain the desired result by writing the above inequality for $h(t)=g(x, y)(t)$. The sharpness of the constants follows by the particular cases which are given in Corollary 1 and Corollary 2. 
Proof of Corollary 1. Choose $s_{0}=0, s_{1}=1$ and $0=\alpha_{0}<\alpha_{1}=s<\alpha_{2}=1$ in Theorem 1 to obtain (3.2). By choosing $s=\frac{1}{2}$ in (3.2), we obtain (3.3). Now, we will prove the sharpness of the constants in (3.3). Let $\alpha$ and $\beta$ be real positive constants such that

$$
\begin{aligned}
& \left|\int_{0}^{1} f[(1-t) x+t y] d t-\frac{f(x)+f(y)}{2}\right| \\
\leq & \left\{\begin{array}{l}
\alpha\|\nabla f[(1-\cdot) x+\cdot y](y-x)\|_{\infty}, \\
\beta\left(\frac{1}{(q+1)^{\frac{1}{q}}}\right)\|\nabla f[(1-\cdot) x+\cdot y](y-x)\|_{p}, \quad p>1, \frac{1}{p}+\frac{1}{q}=1 .
\end{array}\right.
\end{aligned}
$$

Take $X=\mathbb{R},[x, y]=[a, b] \subset \mathbb{R}(a \neq b)$ and $f(x)=\left|x-\frac{b+a}{2}\right|$. Note that $f$ is a convex function on the closed interval $[a, b]$, thus, it is an absolutely continuous function (see [28, Proposition 5.16]). Therefore,

$$
\frac{1}{4}(b-a) \leq\left\{\begin{array}{l}
\alpha(b-a) \\
\beta\left(\frac{1}{(q+1)^{\frac{1}{q}}}\right)(b-a)^{\frac{1}{q}}, q>1 .
\end{array}\right.
$$

From the first case, we obtain $\alpha \geq \frac{1}{4}$ since $b-a \neq 0$, which proves the sharpness of $\frac{1}{4}$ in the first case of (3.3). Now, let $q \rightarrow 1$ in the second case, we obtain $\frac{1}{4}(b-a) \leq \frac{1}{2} \beta(b-a)$, that is, $\beta \geq \frac{1}{2}$, since $b-a \neq 0$, which shows that $\frac{1}{2}$ is sharp in the second case of (3.3).

Now, suppose that

$$
\left|\frac{f(x)+f(y)}{2}-\int_{0}^{1} f[(1-t) x+t y] d t\right| \leq \gamma\|\nabla f[(1-\cdot) x+\cdot y](y-x)\|_{1}
$$

for a real constant $\gamma>0$. By choosing $X=\mathbb{R}$ and the absolutely continuous function $f(x)=\frac{C}{C^{2}+x^{2}}-\tan ^{-1}\left(\frac{1}{C}\right)(C>0)$ on the interval [0,1] (the proof of this part is due to Peachey, McAndrew, and Dragomir [26, p. 99-100]), we obtain

Thus,

$$
\frac{1}{2 C}-\tan ^{-1}\left(\frac{1}{C}\right)+\frac{C}{2\left(C^{2}+1\right)} \leq \gamma\left[\frac{1}{C\left(C^{2}+1\right)}\right]
$$

$$
\gamma \geq\left(C^{2}+1\right)\left[\frac{1}{2}-C \tan ^{-1}\left(\frac{1}{C}\right)+\frac{C^{2}}{2\left(C^{2}+1\right)}\right],
$$

and by taking $C \rightarrow 0^{+}$, we obtain $\gamma \geq \frac{1}{2}$ and the proof for the sharpness of the constants in (3.3) is complete. This implies that all constants in (3.1) and (3.2) are sharp.

Proof of Corollary 2. Choose $s_{0}=0 \leq s_{1}=s \leq 1=s_{2}$ and $0 \leq \alpha_{1} \leq s \leq$ $\alpha_{2} \leq 1$ in Theorem 1 , then let $\alpha_{1}=0$ and $\alpha_{2}=1$, to obtain the (3.4). By choosing $s=\frac{1}{2}$ in (3.4), we obtain (3.5). The proof for the sharpness of the constants in (3.5) is similar to those in (3.3) with the same examples as those in the first two cases and the same choice of function on interval $[-1,1]$ for the third one. This implies that all constants in (3.1) and (3.4) are sharp. 


\section{Application for semi-inner products}

The following result holds in any normed linear space with the semi-inner products $\langle\cdot, \cdot\rangle_{s(i)}$.

Proposition 1. Let $(X,\|\cdot\|)$ be a normed linear space, $I_{k}: 0=s_{0}<s_{1}<$ $\cdots<s_{k-1}<s_{k}=1$ be a division of the interval $[0,1]$ and $\alpha_{i}(i=0, \ldots, k+1)$ be $k+2$ points such that $\alpha_{0}=0, \alpha_{i} \in\left[s_{i-1}, s_{i}\right](i=1, \ldots, k)$ and $\alpha_{k+1}=1$. If $1 \leq r<\infty$ then

$$
\begin{aligned}
& \left|\int_{0}^{1}\|(1-t) x+t y\|^{r} d t-\sum_{i=0}^{k}\left(\alpha_{i+1}-\alpha_{i}\right)\left\|\left(1-s_{i}\right) x+s_{i} y\right\|^{r}\right| \\
& \left\{\begin{array}{l}
{\left[\frac{1}{4} \sum_{i=0}^{k-1} h_{i}^{2}+\sum_{i=0}^{k-1}\left(\alpha_{i+1}-\frac{s_{i}+s_{i+1}}{2}\right)^{2}\right]} \\
\times \sup _{u \in[0,1]}\left[r\|(1-u) x+u y\|^{r-2}\left|\langle y-x,(1-u) x+u y\rangle_{s(i)}\right|\right] \\
\frac{1}{(q+1)^{\frac{1}{q}}}\left[\sum_{i=0}^{k-1}\left[\left(\alpha_{i+1}-s_{i}\right)^{q+1}+\left(s_{i+1}-\alpha_{i+1}\right)^{q+1}\right]\right]^{\frac{1}{q}} \\
\times\left[\int_{0}^{1}\left|r\|(1-u) x+u y\|^{r-2}\langle y-x,(1-u) x+u y\rangle_{s(i)}\right|^{p} d u\right]^{\frac{1}{p}} \\
{\left[\begin{array}{l}
\frac{1}{2} \nu(h)+\max _{i \in\{0, \ldots, k-1\}} \\
\quad
\end{array}\left|\alpha_{i+1}-\frac{s_{i}+s_{i+1}}{2}\right|\right]} \\
\quad \int_{0}^{1}\left|r\|(1-u) x+u y\|^{r-2}\langle y-x,(1-u) x+u y\rangle_{s(i)}\right| d u
\end{array}\right.
\end{aligned}
$$

hold for any $x, y \in X$, whenever $r \geq 2$, otherwise they hold for any linearly independent $x, y \in X$. Here, $\nu(h):=\max \left\{h_{i} \mid i=0, \ldots, k-1\right\}$, and $h_{i}:=$ $s_{i+1}-s_{i}(i=0, \ldots, k-1)$.

Proof. Let $f(x)=\|x\|^{r}$, where $x \in X$, and $1 \leq r<\infty$. Since $f$ is convex on $X$ then $g(x, y)(\cdot)=f((1-\cdot) x+\cdot y)$ is convex on $[0,1]$ for any $1 \leq r<\infty$ and $x, y \in X$. It follows that $g(x, y)(\cdot)=\|(1-\cdot) x+\cdot y\|^{r}$ is an absolutely continuous function. Therefore, we may apply Theorem 1 for $f($ see $(2.1))$ and obtained the desired result.

Remark 4. The result we obtain in Proposition 1 is "complicated" in the sense that the upper bounds are not practical to apply. Here, we suggest simpler, although coarser, upper bounds (see [17, pp. 97-98]) using the Cauchy-Schwarz inequality for semi-inner products. Under the assumptions of Proposition 1, and by Cauchy-Schwarz type inequality for superior (inferior) semi-inner products (see [16, p. 29]), we obtain

$$
\sup _{u \in[0,1]}\left[r\|(1-u) x+u y\|^{r-2}\left|\langle y-x,(1-u) x+u y\rangle_{s(i)}\right|\right.
$$


(5.2) $\leq r\|y-x\| \sup _{u \in[0,1]}\|(1-u) x+u y\|^{r-1}=r\|y-x\| \max \left\{\|x\|^{r-1},\|y\|^{r-1}\right\}$

for all $x, y \in X$.

Note. The last two quantities in (5.2) are equal. The argument is as follows: consider the function $f(t)=\|(1-t) x+t y\|^{r-1}$ on $[0,1]$. Since it is continuous and convex on $[0,1]$, then the supremum of $f$ on $[0,1]$ is exactly its maximum, and it is attained at one of the endpoints.

We also have the following for any $x, y \in X$

$$
\begin{aligned}
& r\left(\int_{0}^{1}\left|\|(1-u) x+u y\|^{r-2}\langle y-x,(1-u) x+u y\rangle_{s(i)}\right|^{p} d u\right)^{\frac{1}{p}} \\
\leq & r\|y-x\|\left(\int_{0}^{1}\|(1-u) x+u y\|^{p(r-1)} d u\right)^{\frac{1}{p}} \\
\leq & r\|y-x\|\left(\frac{\|x\|^{p(r-1)}+\|y\|^{p(r-1)}}{2}\right)^{\frac{1}{p}}
\end{aligned}
$$

by Hermite-Hadamard inequality for the norm (see [21, p. 3] and [27, p. 106]), and

$$
\begin{aligned}
& r\left(\int_{0}^{1}\left|\|(1-u) x+u y\|^{r-2}\langle y-x,(1-u) x+u y\rangle_{s(i)}\right| d u\right) \\
\leq & r\|y-x\|\left(\int_{0}^{1}\|(1-u) x+u y\|^{r-1} d u\right) \\
\leq & \frac{1}{2} r\|y-x\|\left(\|x\|^{r-1}+\|y\|^{r-1}\right),
\end{aligned}
$$

by the refined triangle inequality for the norm (see [21, p. 4] and [27, p. 106]). Therefore, we have the following inequalities

$$
\begin{gathered}
\left|\int_{0}^{1}\|(1-t) x+t y\|^{r} d t-\sum_{i=0}^{k}\left(\alpha_{i+1}-\alpha_{i}\right)\left\|\left(1-s_{i}\right) x+s_{i} y\right\|^{r}\right| \\
(5.3) \leq r\|y-x\|\left\{\begin{array}{c}
{\left[\frac{1}{4} \sum_{i=0}^{k-1} h_{i}^{2}+\sum_{i=0}^{k-1}\left(\alpha_{i+1}-\frac{s_{i}+s_{i+1}}{2}\right)^{2}\right] \sup _{u \in[0,1]}\|(1-u) x+u y\|^{r-1},} \\
\frac{1}{(q+1)^{\frac{1}{q}}}\left[\sum_{i=0}^{k-1}\left[\left(\alpha_{i+1}-s_{i}\right)^{q+1}+\left(s_{i+1}-\alpha_{i+1}\right)^{q+1}\right]\right]^{\frac{1}{q}} \\
\times\left[\int_{0}^{1}\|(1-u) x+u y\|^{p(r-1)} d u\right]^{\frac{1}{p}}, \quad p>1, \frac{1}{p}+\frac{1}{q}=1 ; \\
{\left[\frac{1}{2} \nu(h)+\max _{i \in\{0, \ldots, k-1\}}\left|\alpha_{i+1}-\frac{s_{i}+s_{i+1}}{2}\right|\right] \int_{0}^{1}\|(1-u) x+u y\|^{r-1} d u,}
\end{array}\right.
\end{gathered}
$$


$(5.4) \leq r\|y-x\|\left\{\begin{array}{l}{\left[\frac{1}{4} \sum_{i=0}^{k-1} h_{i}^{2}+\sum_{i=0}^{k-1}\left(\alpha_{i+1}-\frac{s_{i}+s_{i+1}}{2}\right)^{2}\right] \max \left\{\|x\|^{r-1},\|y\|^{r-1}\right\},} \\ \frac{1}{(q+1)^{\frac{1}{q}}}\left[\sum_{i=0}^{k-1}\left[\left(\alpha_{i+1}-s_{i}\right)^{q+1}+\left(s_{i+1}-\alpha_{i+1}\right)^{q+1}\right]\right]^{\frac{1}{q}} \\ \quad \times\left[\frac{\|x\|^{p(r-1)}+\|y\|^{p(r-1)}}{2}\right]^{\frac{1}{p}}, \quad p>1, \frac{1}{p}+\frac{1}{q}=1 ; \\ \frac{1}{2}\left[\frac{1}{2} \nu(h)+\max _{i \in\{0, \ldots, k-1\}} \mid \alpha_{i+1}-\frac{s_{i}+s_{i+1}}{2} \|\right]\left(\|x\|^{r-1}+\|y\|^{r-1}\right),\end{array}\right.$

which hold for any $x, y \in X$. The constants in the first and second cases of (5.3) and (5.4) are sharp. The proof follows by its particular cases which are mentioned in Proposition 2.

Corollary 3. Let $X$ be a normed linear space, $s \in[0,1]$ and $1 \leq r<\infty$. Then, we have the inequalities

$$
\begin{aligned}
& \left|\int_{0}^{1}\|(1-t) x+t y\|^{r} d t-\|(1-s) x+s y\|^{r}\right| \\
& \leq r\|y-x\|\left\{\begin{array}{l}
\left.\left[\frac{1}{4}+\left(s-\frac{1}{2}\right)^{2}\right] \sup _{u \in[0,1]}\|(1-u) x+u y\|^{r-1}\right\}, \\
\frac{1}{(q+1)^{\frac{1}{q}}}\left[s^{q+1}+(1-s)^{q+1}\right]^{\frac{1}{q}}\left(\int_{0}^{1}\|(1-u) x+u y\|^{p(r-1)} d u\right)^{\frac{1}{p}}, \\
{\left[\frac{1}{2}+\left|s-\frac{1}{2}\right|\right]\left(\int_{0}^{1}\|(1-u) x+u y\|^{r-1} d u\right) .}
\end{array}\right. \\
& \leq r\|y-x\|\left\{\begin{array}{l}
{\left[\frac{1}{4}+\left(s-\frac{1}{2}\right)^{2}\right] \max \left\{\|x\|^{r-1},\|y\|^{r-1}\right\},} \\
\frac{1}{(q+1)^{\frac{1}{q}}}\left[s^{q+1}+(1-s)^{q+1}\right]^{\frac{1}{q}}\left(\frac{\|x\|^{p(r-1)}+\|y\|^{p(r-1)}}{2}\right)^{\frac{1}{p}}, \\
\frac{1}{2}\left[\frac{1}{2}+\left|s-\frac{1}{2}\right|\right]\left(\|x\|^{r-1}+\|y\|^{r-1}\right) .
\end{array}\right.
\end{aligned}
$$

for any $x, y \in X$. The constants in the first and second cases of (5.5) and (5.6) are sharp.

Proof. Choose $s_{0}=0 \leq s_{1}=s \leq 1=s_{2}$ and $0 \leq \alpha_{1} \leq s \leq \alpha_{2} \leq 1$, then let $\alpha_{1}=0$ and $\alpha_{2}=1$ in (5.3) and (5.4). The sharpness of the constants follows by the particular case which is pointed out in Proposition 2.

Proposition 2. Particularly, we have

$$
\left|\int_{0}^{1}\|(1-t) x+t y\|^{2} d t-\|(1-s) x+s y\|^{2}\right|
$$


$(5.7) \leq 2\|y-x\|\left\{\begin{array}{l}{\left[\frac{1}{4}+\left(s-\frac{1}{2}\right)^{2}\right] \max \{\|x\|,\|y\|\},} \\ \frac{1}{(q+1)^{\frac{1}{q}}}\left[s^{q+1}+(1-s)^{q+1}\right]^{\frac{1}{q}}\left(\int_{0}^{1}\|(1-u) x+u y\|^{p} d u\right)^{\frac{1}{p}}, \\ {\left[\frac{1}{2}+\left|s-\frac{1}{2}\right|\right]\left(\int_{0}^{1}\|(1-u) x+u y\| d u\right),}\end{array} ;\right.$

$(5.8) \leq 2\|y-x\|\left\{\begin{array}{l}{\left[\frac{1}{4}+\left(s-\frac{1}{2}\right)^{2}\right] \max \{\|x\|,\|y\|\},} \\ \frac{1}{(q+1)^{\frac{1}{q}}\left[s^{q+1}+(1-s)^{q+1}\right]^{\frac{1}{q}}\left(\frac{\|x\|^{p}+\|y\|^{p}}{2}\right)^{\frac{1}{p}},} \\ p>1, \frac{1}{p}+\frac{1}{q}=1 ; \\ \frac{1}{2}\left[\frac{1}{2}+\left|s-\frac{1}{2}\right|\right](\|x\|+\|y\|)\end{array}\right.$

for any $x, y \in X$. The constants in the first and second cases of (5.7) and (5.8) are sharp. We also have

$$
\left|\int_{0}^{1}\|(1-t) x+t y\| d t-\|(1-s) x+s y\|\right| \leq\left[\frac{1}{4}+\left(s-\frac{1}{2}\right)^{2}\right]\|y-x\| .
$$

The constant $\frac{1}{4}$ in (5.9) is sharp.

Proof. We obtain (5.7) and (5.8) by choosing $r=2$ in (5.5) and (5.6), respectively. The proof for the sharpness of the constants is implied by those in Proposition 4). By choosing $r=1$ in (5.5), we obtain

$$
\begin{aligned}
& \left|\int_{0}^{1}\|(1-t) x+t y\| d t-\|(1-s) x+s y\|\right| \\
\leq & \|y-x\|\left\{\begin{array}{l}
\frac{1}{4}+\left(s-\frac{1}{2}\right)^{2}, \\
\frac{1}{(q+1)^{\frac{1}{q}}}\left[s^{q+1}+(1-s)^{q+1}\right]^{\frac{1}{q}}, q>1 ; \\
\frac{1}{2}+\left|s-\frac{1}{2}\right|
\end{array}\right.
\end{aligned}
$$

for any $x, y \in X$. Note that for all $1<q<\infty$ and $s \in[0,1]$,

(1) $\frac{1}{4}+\left(s-\frac{1}{2}\right)^{2}=\frac{s^{2}+(1-s)^{2}}{2}=\int_{0}^{1}|t-s| d t$,

(2) $\frac{1}{(q+1)^{\frac{1}{q}}}\left[s^{q+1}+(1-s)^{q+1}\right]^{\frac{1}{q}}=\left(\int_{0}^{1}|t-s|^{q} d t\right)^{\frac{1}{q}}$,

(3) $\frac{1}{2}+\left|s-\frac{1}{2}\right|=\max \{s, 1-s\}=\sup _{t \in[0,1]}|t-s|$,

and $\int_{0}^{1}|t-s| d t \leq\left(\int_{0}^{1}|t-s|^{q} d t\right)^{\frac{1}{q}} \leq \sup _{t \in[0,1]}|t-s|$ by Hölder inequality. Thus,

$$
\frac{1}{4}+\left(s-\frac{1}{2}\right)^{2} \leq \frac{1}{(q+1)^{\frac{1}{q}}}\left[s^{q+1}+(1-s)^{q+1}\right]^{\frac{1}{q}} \leq \frac{1}{2}+\left|s-\frac{1}{2}\right| .
$$


We conclude that the constant $\frac{1}{4}$ is best possible among the constants in all cases of (5.10) and we obtain (5.9). The proof for the sharpness of the constant will be given in Proposition 4.

\section{Some particular cases of interest}

Proposition 3. Let $X$ be a normed linear space and $1 \leq r<\infty$. Then

$$
\begin{gathered}
0 \leq \int_{0}^{1}\|(1-t) x+t y\|^{r} d t-\left\|\frac{x+y}{2}\right\|^{r} \\
(6.1) \leq r\|y-x\|\left\{\begin{array}{l}
\frac{1}{4} \sup _{u \in[0,1]}\|(1-u) x+u y\|^{r-1}, \\
\frac{1}{2(q+1)^{\frac{1}{q}}}\left(\int_{0}^{1}\|(1-u) x+u y\|^{p(r-1)} d u\right)^{\frac{1}{p}}, p>1, \frac{1}{p}+\frac{1}{q}=1 ; \\
\frac{1}{2} \int_{0}^{1}\|(1-u) x+u y\|^{r-1} d u,
\end{array}\right. \\
(6.2) \leq r\|y-x\|\left\{\begin{array}{l}
\frac{1}{4} \max \left\{\|x\|^{r-1},\|y\|^{r-1}\right\}, \\
\frac{1}{2(q+1)^{\frac{1}{q}}}\left(\frac{\|x\|^{p(r-1)}+\|y\|^{p(r-1)}}{\frac{1}{4}\left(\|x\|^{r-1}+\|y\|^{r-1}\right),}\right)^{\frac{1}{p}}, p>1, \frac{1}{p}+\frac{1}{q}=1 ;
\end{array}\right.
\end{gathered}
$$

hold for any $x, y \in X$. The constants in the first and second cases of (6.1) and (6.2) are sharp.

Proof. Choose $s=\frac{1}{2}$ in (5.5) and (5.6). The sharpness of the constants follows by the particular case which is pointed out in Proposition 4.

Proposition 4. Particularly,

$$
\begin{aligned}
0 \leq \int_{0}^{1}\|(1-t) x+t y\|^{2} d t-\left\|\frac{x+y}{2}\right\|^{2} \\
(6.3) \leq\|y-x\|\left\{\begin{array}{l}
\frac{1}{2} \sup _{u \in[0,1]}\|(1-u) x+u y\|, \\
\frac{1}{(q+1)^{\frac{1}{q}}}\left(\int_{0}^{1}\|(1-u) x+u y\|^{p} d u\right)^{\frac{1}{p}}, p>1, \frac{1}{p}+\frac{1}{q}=1 ; \\
\int_{0}^{1}\|(1-u) x+u y\| d u,
\end{array}\right. \\
(6.4) \leq\|y-x\|\left\{\begin{array}{l}
\frac{1}{2} \max \{\|x\|,\|y\|\}, \\
\frac{1}{(q+1)^{\frac{1}{q}}}\left(\frac{\|x\|^{p}+\|y\|^{p}}{2}\right)^{\frac{1}{p}}, p>1, \frac{1}{p}+\frac{1}{q}=1 ; \\
\frac{1}{2}(\|x\|+\|y\|),
\end{array}\right.
\end{aligned}
$$

hold for any $x, y \in X$. The constants $\frac{1}{2}$ and 1 are sharp in the first and second cases of (6.3) and (6.4). We also have

$$
0 \leq \int_{0}^{1}\|(1-t) x+t y\| d t-\left\|\frac{x+y}{2}\right\| \leq \frac{1}{4}\|y-x\| .
$$


The constant $\frac{1}{4}$ in (6.5) is the best possible constant.

Proof. We obtain (6.3) and (6.4) by choosing $r=2$ in (6.1) and (6.2), respectively. Now we will prove the sharpness of the constants in the first two cases. Suppose that the inequality holds for the constant $A>0$ instead of $\frac{1}{2}$, that is,

$$
\int_{0}^{1}\|(1-t) x+t y\|^{2} d t-\left\|\frac{x+y}{2}\right\|^{2} \leq A\|y-x\| \max \{\|x\|,\|y\|\} .
$$

Note that it is sufficient for us to prove the sharpness of the constant in the first case of $(6.4)$, since both quantities are equal. Choose $(X,\|\cdot\|)=\left(\mathbb{R}^{2},\|\cdot\|_{1}\right)$, $x=\left(\frac{1}{n}, n\right)$, and $y=\left(-\frac{1}{n}, n\right)$ for $n \in \mathbb{N}$, then we have

$$
\frac{3 n^{2}+1}{3 n^{2}} \leq A\left(\frac{2 n^{2}+2}{n^{2}}\right) \text {. }
$$

Taking $n \rightarrow \infty$, we obtain $1 \leq 2 A$, that is, $A \geq \frac{1}{2}$.

Note that the constants in the second case of (6.3) and (6.4) are also sharp. Suppose that the inequality holds for the constants $B, C>0$ instead of the multiplicative constant 1 , that is,

$$
\begin{aligned}
& \int_{0}^{1}\|(1-t) x+t y\|^{2} d t-\left\|\frac{x+y}{2}\right\|^{2} \\
\leq & B \frac{\|y-x\|}{(q+1)^{\frac{1}{q}}}\left(\int_{0}^{1}\|(1-u) x+u y\|^{p} d u\right)^{\frac{1}{p}} \leq C \frac{\|y-x\|}{(q+1)^{\frac{1}{q}}}\left(\frac{\|x\|^{p}+\|y\|^{p}}{2}\right)^{\frac{1}{p}} .
\end{aligned}
$$

Choose $(X,\|\cdot\|)=\left(\mathbb{R}^{2},\|\cdot\|_{1}\right), x=\left(\frac{1}{n}, n\right)$, and $y=\left(-\frac{1}{n}, n\right)$ for $n \in \mathbb{N}$, then we have

$$
\frac{3 n^{2}+1}{3 n^{2}} \leq 2 B\left(\frac{\left(n^{2}\left(n^{2}+1\right)^{p}+\left(n^{2}+1\right)^{p}-n^{2 p+2}\right)^{\frac{1}{p}}}{n^{2}(q+1)^{\frac{1}{q}}(p+1)^{\frac{1}{p}}}\right) \leq C\left(\frac{2\left(n^{2}+1\right)}{n^{2}(q+1)^{\frac{1}{q}}}\right) .
$$

Taking $q \rightarrow 1$ and $n \rightarrow \infty$, we obtain $B \geq 1$ and $C \geq 1$.

By choosing $r=1$ in (6.1) (or (6.2)), we obtain

$$
0 \leq \int_{0}^{1}\|(1-t) x+t y\| d t-\left\|\frac{x+y}{2}\right\| \leq\left\{\begin{array}{l}
\frac{1}{4}\|y-x\|, \\
\frac{1}{2(q+1)^{\frac{1}{q}}}\|y-x\|, q>1 \\
\frac{1}{2}\|y-x\|
\end{array}\right.
$$

for any $x, y \in X$. Note that for any $1<q<\infty$, we have $\frac{1}{4} \leq \frac{1}{2(q+1)^{\frac{1}{q}}} \leq \frac{1}{2}$ (the proof follows by choosing $s=\frac{1}{2}$ in $(5.11)$ ). Therefore, $\frac{1}{4}$ is the best possible among the constants of all cases in (6.6) and we get (6.5). Now, suppose that the inequality holds for any constant $D>0$ instead of $\frac{1}{4}$, that is,

$$
\int_{0}^{1}\|(1-t) x+t y\| d t-\left\|\frac{x+y}{2}\right\| \leq D\|y-x\| .
$$

Choose $(X,\|\cdot\|)=\left(\mathbb{R}^{2},\|\cdot\|_{1}\right), x=(2,1)$, and $y=(2,-1)$ to obtain $\frac{1}{2} \leq 2 D$, that is, $D \geq \frac{1}{4}$. Thus, the constant $\frac{1}{4}$ is sharp. 
Remark 5 (The case of inner product space). If $X$ is an inner product space, the constant in the first case of (6.4) is not sharp, since

$$
\int_{0}^{1}\|(1-t) x+t y\|^{2} d t-\left\|\frac{x+y}{2}\right\|^{2}=\frac{1}{12}\|y-x\|^{2},
$$

and the fact that

$$
\frac{1}{12}\|y-x\|^{2} \leq \frac{1}{12}\|y-x\|(\|x\|+\|y\|) \leq \frac{1}{6}\|y-x\| \max \{\|x\|,\|y\|\} .
$$

The sharpness of the constant in the second case of (6.4) is not preserved in this case, since we have the fact that

$$
\frac{1}{12}\|y-x\|^{2} \leq \frac{1}{12}\|y-x\|(\|x\|+\|y\|) \leq \frac{1}{6}\|y-x\|\left(\|x\|^{p}+\|y\|^{p}\right)^{\frac{1}{p}},
$$

and that $\frac{1}{6} \leq \frac{1}{2^{\frac{1}{p}}(q+1)^{\frac{1}{q}}}$. The constant in the third case of (6.4) is not sharp, since

$$
\frac{1}{12}\|y-x\|^{2} \leq \frac{1}{12}\|y-x\|(\|x\|+\|y\|) .
$$

Similarly, for the trapezoidal functionals, we have the following facts:

$$
\frac{\|x\|^{2}+\|y\|^{2}}{2}-\int_{0}^{1}\|(1-t) x+t y\|^{2} d t=\frac{1}{6}\|y-x\|^{2},
$$

and

(1) $\frac{1}{6}\|y-x\|^{2} \leq \frac{1}{6}\|y-x\|(\|x\|+\|y\|) \leq \frac{1}{3}\|y-x\| \max \{\|x\|,\|y\|\} ;$

(2) $\frac{1}{6}\|y-x\|^{2} \leq \frac{1}{6}\|y-x\|(\|x\|+\|y\|) \leq \frac{1}{3}\|y-x\|\left(\|x\|^{p}+\|y\|^{p}\right)^{\frac{1}{p}}$ and that $\frac{1}{3} \leq \frac{1}{2^{\frac{1}{p}}(q+1)^{\frac{1}{q}}}$

(3) $\frac{1}{6}\|y-x\|^{2} \leq \frac{1}{6}\|y-x\|(\|x\|+\|y\|) ;$

which show that the constants in these upper bounds are not sharp for the trapezoidal cases.

The constant $\frac{1}{4}$ in (6.5) (and also in the trapezoidal case) remains sharp in this case. The proof follows by choosing $(X,\|\cdot\|)=(\mathbb{R},|\cdot|), x=1$, and $y=-1$.

\section{Comparison analysis}

In $[21$, p. 11, 15], we considered an Ostrowski type inequality for convex functions on linear spaces and obtained the following result in any normed space $(X,\|\cdot\|)$

$\int_{0}^{1}\|(1-t) x+t y\|^{r} d t-\left\|\frac{x+y}{2}\right\|^{r} \leq \frac{1}{8} r\left(\left\langle y-x, y\|y\|^{r-2}\right\rangle_{i}-\left\langle y-x, x\|x\|^{r-2}\right\rangle_{s}\right)$

for any $x, y \in X$ whenever $r \geq 2$; otherwise they hold for nonzero $x, y \in X$, and $\langle\cdot, \cdot\rangle_{s(i)}$ is the superior (inferior) semi-inner product with respect to the norm $\|\cdot\|$.

In this paper, we have considered the Ostrowski type inequality for absolutely continuous functions, which is more general than [21] and have obtained 
the following bounds for the left-hand side of the inequalities above (see (6.1) and $(6.2))$. The bound that we have obtained is,

$$
\frac{1}{4} r\|y-x\| \sup _{u \in[0,1]}\|(1-u) x+u y\|^{r-1}=\frac{1}{4} r\|y-x\| \max \left\{\|x\|^{r-1},\|y\|^{r-1}\right\} .
$$

Note. Since the last two bounds in (6.1) and (6.2) are not significant for the case of $r=1$ (see Proposition 4), we consider only the first bound for this section.

We want to compare the two bounds: $\frac{1}{8} r\left(\left\langle y-x, y\|y\|^{r-2}\right\rangle_{i}-\left\langle y-x, x\|x\|^{r-2}\right\rangle_{s}\right)$ and $\frac{1}{4} r\|y-x\| \max \left\{\|x\|^{r-1},\|y\|^{r-1}\right\}$. The bound that we obtained in this paper is simpler in the sense that it only involves the given norm, while the other one involves not only the given norm, but also the superior (inferior) semi-inner product associated to the norm. However, the bounds in [21] are proven better for the case of inner product spaces, where $r=1$ and $r=2$. The verification is as follows:

Case of $\mathbf{r}=1$. We wish to compare $\frac{1}{8}\left\langle y-x, \frac{y}{\|y\|}-\frac{x}{\|x\|}\right\rangle$ and $\frac{1}{4}\|y-x\|$ (for nonzero $x, y \in X)$. We recall the Dunkl-Williams inequality (see [20, p. 53], $[22$, p. 890] and [23, p. 448])

$$
\left\|\frac{x}{\|x\|}-\frac{y}{\|y\|}\right\| \leq \frac{2\|x-y\|}{\|x\|+\|y\|},
$$

which holds for nonzero $x$ and $y$ in an inner product space $X$. Now, for $x, y \in X$ where $x, y \neq 0$, we have

$$
\begin{aligned}
\frac{1}{8}\left\langle y-x, \frac{y}{\|y\|}-\frac{x}{\|x\|}\right\rangle & \leq \frac{1}{8}\|y-x\|\left\|\frac{y}{\|y\|}-\frac{x}{\|x\|}\right\| \\
& \leq \frac{1}{4}\|y-x\| \frac{\|x-y\|}{\|x\|+\|y\|} \\
& \leq \frac{1}{4}\|y-x\| \frac{\|x\|+\|y\|}{\|x\|+\|y\|}=\frac{1}{4}\|y-x\| .
\end{aligned}
$$

We conclude that the bound in [21] are better.

Case of $\mathbf{r}=2$. We want to compare $\frac{1}{4}\langle y-x, y-x\rangle=\frac{1}{4}\|y-x\|^{2}$ and $\frac{1}{2}\|y-x\| \max \{\|x\|,\|y\|\}$. For all $x, y \in X$, we have

$$
\frac{1}{4}\|y-x\|^{2} \leq \frac{1}{4}\|y-x\|(\|x\|+\|y\|) \leq \frac{1}{2}\|y-x\| \max \{\|x\|,\|y\|\} .
$$

We conclude that the bound in [21] are better.

Case of $1<r<\infty, r \neq 2$. We conjecture that

Conjecture 1. In an inner product space $(X,\langle\cdot, \cdot\rangle)$, the following inequality

$$
\frac{1}{8} r\left\langle y-x, y\|y\|^{r-2}-x\|x\|^{r-2}\right\rangle \leq \frac{1}{4} r\|y-x\| \max \left\{\|x\|^{r-1},\|y\|^{r-1}\right\}
$$


holds for any $x, y \in X$ whenever $r \geq 2$; otherwise it holds for any nonzero $x, y \in X$.

We observe that the above statement is true in some cases. Taking $X=\mathbb{R}$ and multiplication as its inner product and utilizing MAPLE for the following functions

$$
\Phi(x, y):=\frac{1}{4} r|y-x| \max \left\{|x|^{r-1},|y|^{r-1}\right\}-\frac{1}{8} r(y-x)\left(y|y|^{r-2}-x|x|^{r-2}\right)
$$

for $x, y \in \mathbb{R}$, we observe that for several values of $r$, we have $\Phi(x, y) \geq 0$ for any $x, y \in X$ (see Figure 1 for the plot of $\Phi$ with the choice of $r=3$ ). However, we have no analytical proof for this statement.

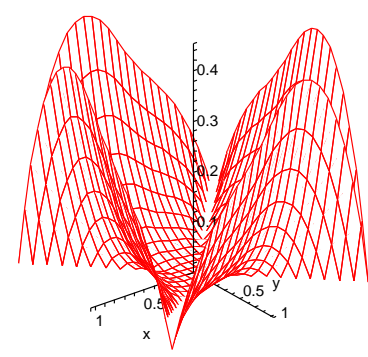

(a) Plot of $\Phi$ for $r=3$.

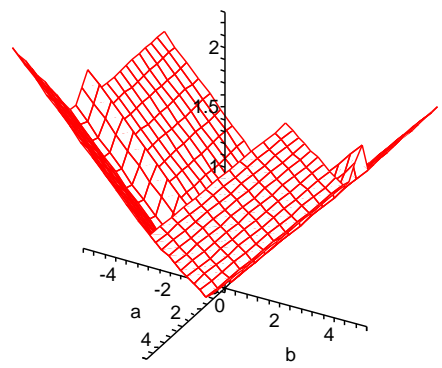

(b) Plot of $\Psi$.

FIGURE 1

Conjecture 2. In a normed linear space $(X,\|\cdot\|)$, the following inequality $\frac{1}{8} r\left(\left\langle y-x, y\|y\|^{r-2}\right\rangle_{i}-\left\langle y-x, x\|x\|^{r-2}\right\rangle_{s}\right) \leq \frac{1}{4} r\|y-x\| \max \left\{\|x\|^{r-1},\|y\|^{r-1}\right\}$

holds for any $x, y \in X$ whenever $r \geq 2$; otherwise it holds for any nonzero $x, y \in X$ (here, $\langle\cdot, \cdot\rangle_{s(i)}$ is the superior (inferior) semi-inner product respect to the norm $\|\cdot\|)$.

We observe that the above statement is true in some cases. Taking $(X,\|\cdot\|)=$ $\left(\mathbb{R}^{2},\|\cdot\|_{1}\right)$ and consider the case of $r=1$, we have the functions

$$
\begin{aligned}
f(x, y) & :=\frac{1}{8}\left[\sum_{y_{i} \neq 0} \frac{y_{i}}{\left|y_{i}\right|}\left(y_{i}-x_{i}\right)-\sum_{y_{i}=0}\left|y_{i}-x_{i}\right|-\sum_{x_{i} \neq 0} \frac{x_{i}}{\left|x_{i}\right|}\left(y_{i}-x_{i}\right)-\sum_{x_{i}=0}\left|y_{i}-x_{i}\right|\right] \\
g(x, y) & :=\frac{1}{4}\|y-x\|_{1}
\end{aligned}
$$


for $x, y \in \mathbb{R}^{2}$. We observe that $f(x, y) \leq g(x, y)$ for some $x, y \in X$ (We choose $x=(1,0)$ and $y=(a, b)(a, b \neq 0)$ and plot the non negative function $\Psi(a, b):=g(x, y)-f(x, y)=\frac{1}{4}(|a-1|+|b|)-\frac{1}{8}\left(\frac{a(a-1)}{|a|}+\frac{b^{2}}{|b|}-(a-1)-|b|\right)$ in Figure 1). However, we do not have an analytical proof for this statement.

\section{References}

[1] C. D. Aliprantis and O. Burkinshaw, Principles of Real Analysis, North-Holland Publishing Co., New York, 1981.

[2] G. A. Anastassiou, Ostrowski type inequalities, Proc. Amer. Math. Soc. 123 (1995), no. $12,3775-3781$.

[3] N. S. Barnett, C. Buşe, P. Cerone, and S. S. Dragomir, Ostrowski's inequality for vectorvalued functions and applications, Comput. Math. Appl. 44 (2002), no. 5-6, 559-572.

[4] P. Cerone, Three point rules in numerical integration, Nonlinear Anal. 47 (2001), no. 4, $2341-2352$.

[5] _ A new Ostrowski type inequality involving integral means over end intervals, Tamkang J. Math. 33 (2002), no. 2, 109-118.

[6] _ On relationships between Ostrowski, trapezoidal and Chebychev identities and inequalities, Soochow J. Math. 28 (2002), no. 3, 311-328.

[7] P. Cerone and S. S. Dragomir, Three point identities and inequalities for $n$-time differentiable functions, SUT J. Math. 36 (2000), no. 2, 351-383.

[8] _ On some inequalities arising from Montgomery's identity (Montgomery's identity), J. Comput. Anal. Appl. 5 (2003), no. 4, 341-367.

[9] P. Cerone, S. S. Dragomir, and J. Roumeliotis, Some Ostrowski type inequalities for n-time differentiable mappings and applications, Demonstratio Math. 32 (1999), no. 4, 697-712.

[10] S. S. Dragomir, The Ostrowski integral inequality for mappings of bounded variation, Bull. Austral. Math. Soc. 60 (1999), no. 3, 495-508.

[11] A generalization of Ostrowski integral inequality for mappings whose derivatives belong to $L_{1}[a, b]$ and applications in numerical integration, J. Comput. Anal. Appl. 3 (2001), no. 4, 343-360.

[12] A generalization of the Ostrowski integral inequality for mappings whose derivatives belong to $L_{p}[a, b]$ and applications in numerical integration, J. Math. Anal. Appl. 255 (2001), no. 2, 605-626.

[13] An inequality improving the first Hermite-Hadamard inequality for convex functions defined on linear spaces and applications for semi-inner products, JIPAM. J. Inequal. Pure Appl. Math. 3 (2002), no. 2, Article 31, 8 pp.

[14] _ An inequality improving the second Hermite-Hadamard inequality for convex functions defined on linear spaces and applications for semi-inner products, JIPAM. J. Inequal. Pure Appl. Math. 3 (2002), no. 3, Article 35, 8 pp.

[15] An Ostrowski like inequality for convex functions and applications, Rev. Mat. Complut. 16 (2003), no. 2, 373-382.

[16] , Semi-inner Products and Applications, Nova Science Publishers, Inc., Hauppauge, NY, 2004.

[17] _ Ostrowski type inequalities for functions defined on linear spaces and applications for semi-inner products, J. Concr. Appl. Math. 3 (2005), no. 1, 91-103.

[18] _ An Ostrowski type inequality for convex functions, Univ. Beograd. Publ. Elektrotehn. Fak. Ser. Mat. 16 (2005), 12-25.

[19] S. S. Dragomir and T. M. Rassias, Generalisations of the Ostrowski Inequality and Applications, Kluwer Acad. Publ., Dordrecht, 2002. 
[20] C. F. Dunkl and K. S. Williams, Mathematical notes: a simple norm inequality, Amer. Math. Monthly 71 (1964), no. 1, 53-54.

[21] E. Kikianty, S. S. Dragomir, and P. Cerone, Sharp inequalities of Ostrowski type for convex functions defined on linear spaces and applications, Comput. Math. Appl., to appear.

[22] W. A. Kirk and M. F. Smiley, Mathematical notes: another characterization of inner product spaces, Amer. Math. Monthly 71 (1964), no. 8, 890-891.

[23] P. R. Mercer, The Dunkl-Williams inequality in an inner product space, Math. Inequal. Appl. 10 (2007), no. 2, 447-450.

[24] D. S. Mitrinović, J. E. Pečarić, and A. M. Fink, Inequalities Involving Functions and Their Integrals and Derivatives, Mathematics and its Applications (East European Series), vol. 53, Kluwer Academic Publishers Group, Dordrecht, 1991.

[25] A. Ostrowski, Über die Absolutabweichung einer differentiebaren Funktion von ihrem Integralmittelwert, Comment. Math. Helv. 10 (1938), 226-227.

[26] T. C. Peachey, A. Mcandrew, and S. S. Dragomir, The best constant in an inequality of Ostrowski type, Tamkang J. Math. 30 (1999), no. 3, 219-222.

[27] J. E. Pečarić and S. S. Dragomir, A generalization of Hadamard's inequality for isotonic linear functionals, Rad. Mat. 7 (1991), no. 1, 103-107.

[28] H. L. Royden, Real Analysis, second ed., Macmillan Publishing Co. Inc., New York, 1968.

EDER KIKIANTY

Research Group of Mathematical Inequalities and Applications SCHOOL OF ENGINEERING AND SCIENCE

VICTORIA UNIVERSITY

PO Box 14428, Melbourne City MC, Victoria 8001, Australia

E-mail address: eder.kikianty@vu.edu.au

SEVER S. Dragomir

Research Group of Mathematical Inequalities and Applications

School of EngineEring And Science

VICTORIA UNIVERSITY

PO Box 14428, Melbourne City MC, Victoria 8001, Australia

E-mail address: Sever.Dragomir@vu.edu.au

Pietro Cerone

Research Group of Mathematical Inequalities and Applications

School of EngineEring And ScIEnCE

VICTORIA UNIVERSITY

PO Box 14428, Melbourne City MC, Victoria 8001, Australia

E-mail address: Pietro.Cerone@vu.edu.au 